\title{
Condiciones de la innovación pecuaria y producción de lácteos en comunidades altoandinas
}

\section{Conditions of livestock and dairy production innovations in high Andean villages}

Diana Rosas Morales ${ }^{1} \&$ Ruth García Pacheco ${ }^{1}$

${ }^{1}$ Universidad Nacional de San Antonio Abad del Cusco-Perú

Autor de correspondencia:diana.rosas@unsaac.edu.pe

\section{Resumen}

El estudio explora las condiciones de los cambios ocurridos en la actividad pecuaria del de dos comunidades del sur andino de Perú, a partir de la innovación en los procesos de producción de ganado vacuno para la obtención de leche. Se utilizó una metodología mixta que involucró el desarrollo de entrevistas y encuestas con las familias de productores del distrito de Ocongate. Se evidencia el incremento de cabezas y criadores de vacunos, mejoras de la producción de leche, incremento de los ingresos familiares, así como otros cambios en la vida familiar de los productores. Además, se argumenta que la participación de instituciones gubernamentales y privadas coadyuvó a la innovación pecuaria.

Palabras clave: innovación pecuaria, lácteos, ganadería, Andes

\begin{abstract}
The study explores the conditions of the changes that have occurred in the livestock activity of two communities in the southern Andes of Peru, based on innovation in the processes of cattle production for obtaining milk. A mixed methodology was used that involved the development of interviews and surveys with the families of producers in the district of Ocongate. The results show an increase in the number of heads of cattle and cattle breeders, improvements in milk production, an increase in family income and other changes in the family life of producers. In addition, it is argued that the involvement of both governmental and private institutions contributed to livestock innovation.
\end{abstract}

Keywords: livesstock innovation, dairy production, breeding, Andes

\section{Introducción}

Entre los años 1990 y 2011, se registró el incremento significativo en la producción 
de leche vacuna en las regiones andinas de Perú, Bolivia y Ecuador. La producción se incrementó hasta 4 veces en estos países, mientras a nivel mundial el crecimiento en la obtención de leche solo fue de 0.4 (Aubron, 2014). En Perú, una serie de factores se combinaron para coadyuvar al impulso de la ganadería vacuna, como la intervención de organismos de cooperación internacional, las reformas políticas en favor de los gobiernos locales y la mejora de vías de comunicación, entre otros.

En las unidades productivas familiares, múltiples innovaciones han posibilitado el desarrollo de la ganadería lechera, principalmente en aspectos como los procesos de crianza del ganado vacuno y la obtención de leche de calidad. La introducción de estas innovaciones demuestra que las poblaciones rurales son proclives al cambio y al riesgo, lo que contradice la literatura convencional que aún las catalogan como "adversas al riesgo" (Touzard, 2018).

En tal perspectiva, nos interesamos en conocer los elementos asociados a las innovaciones en la cadena de producción de ganado vacuno a partir del caso de Ocongate (Perú), donde la actividad pecuaria se ha reorientado a este tipo de ganadería, luego de haber estado centrada en la crianza de alpacas y ovinos (Rosas, 2015; Casallo, 2015). El presente estudio exploró las condiciones de la innovación en la crianza de ganado vacuno y producción de lácteos en dos comunidades andinas de Ocongate, así como las sinergias que las hicieron posible y los cambios asociados en la vida de las familias de los productores.

Los principales resultados evidencian significativas innovaciones en la cadena productiva, traducidas en los incrementos en la cantidad de criadores y cabezas de vacunos de raza, las tierras cultivadas con pastos mejorados y los volúmenes de leche orientados al mercado. El rendimiento promedio de leche por vacuno pasó de 3.25 a 7 litros al día entre los años 2006 y 2013 (Casallo, 2015). La mayoría de la leche se dirige a las plantas transformadoras modernas que elaboran quesos y otros alimentos comerciados en la región. Las familias han dirigido sus mayores ingresos económicos a la educación de los hijos, a mejoras en las condiciones de sus viviendas, acceso a servicios básicos, aparatos tecnológicos y vehículos motorizados. El ser emprendedor ha pasado a ser un logro valorado por la comunidad, junto al acceso a la educación superior.

\section{Métodos}

Seguimos un enfoque mixto que combinó data cualitativa y cuantitativa. Realizamos el trabajo de campo en Ocongate (distrito de la provincia de Quispicanchi y departamento de Cusco. La información presentada fue recogida en dos comunidades: Andamayo y Ccoñamuro, entre los meses de octubre de 2018 y diciembre de 2019. Una de las primeras actividades fue la asistencia a las asambleas comunales para socializar los objetivos de la investigación y el consentimiento informado de los productores para su participación en calidad de informantes. Los consentimientos informados fueron refrendados por los dos presidentes comunales respectivos.

El componente cualitativo consistió en entrevistas a las autoridades comunales, 
criadores de ganado vacuno, productores de queso, funcionarios municipales y otros actores locales. Asimismo, realizamos un grupo focal con productores de ganado vacuno y criadores de cuyes de ambas comunidades. Las entrevistas se realizaron en quechua y castellano, y fueron grabadas previo consentimiento de los informantes.

Para el componente cuantitativo, aplicamos encuestas a varones y mujeres de ambas comunidades. Se calculó una muestra probabilística en base a un total de 145 familias (75 en Andamayo y 70 en Ccoñamuro), información proporcionada por las autoridades comunales. El resultado fue una muestra de 51 familias (con un grado de confianza del 95\%). Las encuestas se procesaron en el programa SPSS.

Por último, revisamos bibliografía relacionada con el tema, información de los Censos Nacionales de Población y vivienda, así como de los dos últimos Censos Nacionales Agropecuarios (Cenagro) ejecutados por el Instituto Nacional de Estadística e Informática (INEI).

\section{Resultados}

La proporción de la población económicamente activa (PEA) del distrito de Ocongate que se dedica a actividades agropecuarias se ha reducido en las últimas décadas: en 1993, el 67\% tenía como principal actividad a la agricultura; y en 2017, el porcentaje se redujo al 59\% (INEI, 1993; 2017). En contraste a la reducción de la PEA agropecuaria, el empleo en industrias manufactureras se ha incrementado de $1.6 \%$ en 1993 a 4.6\% en 2017 (ver tabla 1). Esto corresponde a la transformación de lácteos, particularmente en la fabricación de quesos en Andamayo y Coñamuro; y de confección de tejidos a partir de la fibra de alpaca en las zonas ecológicas más altas. Otro dato significativo es el de los servicios colaterales a la manufactura, como comercios, restaurantes y alojamientos, que pasó de $10.5 \%$ el año 1993 a $18.8 \%$ en 2017 (INEI, 1993; 2017). 
Tabla 1.

PEA y estructura por actividad económica de Ocongate: 1993, 2007 y 2017

\begin{tabular}{|c|c|c|c|c|c|c|}
\hline \multirow{2}{*}{ Actividad económica } & \multicolumn{2}{|c|}{ CENSO 1993} & \multicolumn{2}{|c|}{ CENSO 2007} & \multicolumn{2}{|c|}{ CENSO 2017} \\
\hline & $\mathrm{N}^{\circ}$ & $\%$ & $\mathrm{~N}^{\circ}$ & $\%$ & $\mathrm{~N}^{\circ}$ & $\%$ \\
\hline $\begin{array}{l}\text { Agricultura, ganadería } \\
\text { Explotación de minas }\end{array}$ & 1674 & $67.9 \%$ & 3002 & $66.7 \%$ & 2283 & $59 \%$ \\
\hline y canteras & 4 & $0.2 \%$ & 35 & $0.8 \%$ & 44 & $1.1 \%$ \\
\hline Industrias manufactureras & 39 & $1.6 \%$ & 133 & $2.9 \%$ & 178 & $4.6 \%$ \\
\hline $\begin{array}{l}\text { Construcción, electricidad, } \\
\text { agua y desagüe }\end{array}$ & 17 & $0.7 \%$ & 399 & $8.9 \%$ & 181 & $4.7 \%$ \\
\hline Servicios & 259 & $10.5 \%$ & 574 & $12.7 \%$ & 727 & $8.8 \%$ \\
\hline Otras y no especificados & 326 & $13.2 \%$ & 240 & $5.3 \%$ & 113 & $2.9 \%$ \\
\hline Desocupados & 145 & $5.9 \%$ & 121 & $2.7 \%$ & 342 & $8.8 \%$ \\
\hline Total & 2464 & $100 \%$ & 4504 & $100 \%$ & 3868 & $100 \%$ \\
\hline
\end{tabular}

Fuente: Elaboración propia a partir de los Censos nacionales de población y vivienda 1993, 2007 y 2017 (INEI).

La unidad agropecuaria está constituida por la familia, en Andamayo y Coñamuro se conforma por 4 a 5 personas, en promedio. Algo más de la mitad de las familias (51\%) tiene 2 hijos o menos. Se observa una predominancia a identificar al padre como el jefe de la familia ( $81 \%$ de encuestados), mientras solo el $17 \%$ consideran a la madre como la jefa del hogar.

Las innovaciones se han dado en varias etapas de la cadena productiva, la que en Ocongate, está dirigida a la obtención de leche de vaca y sus derivados. La cadena inicia con la crianza del ganado vacuno, caracterizada por el manejo de pequeños hatos (el 96\% de criadores cuenta con menos de 10 cabezas de ganado). Según los datos de los Cenagros de 1994 y 2012, en el periodo inter-censal el número de animales de tipo vacuno casi se ha duplicado, principalmente de raza Brown-swiss, así como de familias dedicadas a su crianza (ver tabla 2). Los criadores pasaron del $4.6 \%$ al $52.7 \%$ en 2012 ; mientras que el número de cabezas vacunas de raza pasó del $7 \%$ del total del distrito en 1994, al 56.3\% en el año 2012. 
Tabla 2.

Productores y población de ganado vacuno en el distrito de Ocongate entre 1994 y 2012

\begin{tabular}{lcccccc}
\hline & \multicolumn{3}{c}{1994} & & \multicolumn{3}{c}{2012} & \\
& Total & De raza & $\%$ & Total & De raza & $\%$ \\
\hline $\begin{array}{l}\text { Cantidad de } \\
\text { ganado vacuno }\end{array}$ & 3543 & 252 & 7.1 & 6929 & 3904 & 56.3 \\
$\begin{array}{l}\text { Unidades } \\
\text { productivas }\end{array}$ & 1061 & 49 & 4.6 & 1955 & 1946 & 52.7 \\
\hline
\end{tabular}

Fuente: Elaboración propia en base a III Cenagro 1994, IV Cenagro 2012 (INEI).

En Ccoñamuro y Andamayo, el $68 \%$ de las tierras se destinan a la ganadería (entre crianza y cultivo de pastos) y solo el $32 \%$ a la agricultura de autoconsumo. Los pastizales se han extendido significativamente debido a la introducción de semillas y al mejoramiento e implementación de sistemas de riego por varias entidades gubernamentales.

La producción de leche tiene dos destinos: los consumidores locales de leche fresca y las plantas transformadoras. La mayoría de productores prefiere vender la leche a estas últimas, las que verifican las condiciones óptimas de calidad (ph y densidad de la leche). El rendimiento de leche por vaca se elevó de 3.25 litros por día a casi 7 litros por día entre los años 2006 y 2013. Aunque el dato real actualizado debería pasar por un seguimiento registrado, las versiones verbales de los técnicos de la Municipalidad de Ocongate indican que al 2019 se llega a un promedio de 10 litros/ día por vaca.

Respecto a la transformación de la leche, al año 2019 funcionaban 18 plantas transformadoras en todo el distrito; de las cuales, una era administrada comunalmente y las otras 17 eran familiares. Las plantas producen 1,3 toneladas promedio de queso al día, que equivale a 72 kilos de queso al día por planta ${ }^{1}$. En las visitas de campo, observamos una especialización en los productos, en queso paria, andino y/o yogurt, entre otros.

La estructura de los ingresos familiares nos permite acercarnos a las repercusiones de las innovaciones en los hogares. Asencio y Trivelli (2011) encontraron que las fuentes de ingresos de los hogares de Ocongate son diversas, y cada familia cuenta con entre 2 y 3 actividades generadoras de ingresos (p. 22). En la tabla 3, comparamos los datos registrados por Asencio y Trivelli (2011) con los encontrados durante nuestro trabajo de campo. Se observa una reducción de la participación de las actividades primarias como fuente de ingresos en el periodo 1985-2010, y su posterior recuperación para el año 2019. Los ingresos por servicios siguen en ascenso; mientras se reduce el trabajo asalariado, ya que las personas prefieren dedicarse a actividades relacionadas a la producción ganadera. 
Tabla 3.

Evolución de la estructura de ingresos de las familias de Ocongate: 1985-2019 (expresado en porcentajes)

\begin{tabular}{lcccc}
\hline \multicolumn{1}{c}{ Fuente } & \multicolumn{3}{c}{ AÑO } \\
& 1985 & 2000 & 2010 & 2019 \\
\hline Actividad primaria y derivados & 53.7 & 46 & 26.5 & 31 \\
Asalariados & 28.5 & 30.2 & 36.1 & 29 \\
Servicios & 15.5 & 21.4 & 25.2 & 27 \\
Transferencias & 0 & 0 & 7.4 & 6 \\
Artesanía y otras actividades & 2.3 & 2.4 & 4.8 & 7 \\
\hline
\end{tabular}

Fuente: Elaboración propia en base a la encuesta del IEP-2010 (Asencio y Trivelli, 2010) y trabajo campo 2019.

Otro indicador de la mejora en los ingresos familiares lo constituyen las características de las viviendas y la estructura de gastos. La paja utilizada tradicionalmente para el techo de las casas está siendo reemplazada por calaminas y tejas, y algunas familias han empezado a construir sus casas con concreto en lugar de adobe. Además, casi la totalidad de las familias de Ccoñamuro y Andamayo cuentan con agua potable y electricidad en sus domicilios (ver tabla 4). Los gastos familiares en educación y en vivienda se incrementaron. Igualmente, son importantes los gastos en transporte, comunicaciones (celulares), adquisición de artefactos y vehículos. En los últimos 10 años, las familias que cuentan con al menos una motocicleta, ha pasado del $4 \%$ al $56 \%$.

Tabla 4.

Características de la vivienda y acceso a servicios básicos en Ccoñamuro y Andamayo entre 2003-2018

\section{Características de las viviendas $2008 \quad 2018$ y servicios básicos}

Casa de adobe y techo de paja

Casa de adobe y techo de calamina

Adobe y teja

Casa de concreto y teja

Agua potable en domicilio

Energía eléctrica en domicilio

Servicios higiénicos en domicilio

$\begin{array}{cc}27 \% & 8 \% \\ 29 \% & 44 \% \\ 44 \% & 44 \% \\ -- & 2 \% \\ 28 \% & 98 \% \\ 24 \% & 100 \% \\ 4 \% & 94 \%\end{array}$

Fuente: Elaboración propia en base a trabajo de campo 2018-2019. 


\section{Discusión}

En términos generales, la innovación se refiere a un proceso de múltiples etapas que permite a las organizaciones transformar las ideas en nuevos y/o mejorados productos, servicios o procesos, con el fin de mejorar su competitividad y resaltar en el mercado (Baregheh et al., 2009). Durante el siglo XX, la innovación ha sido implícita y, más tarde, explícitamente parte de la idea de crecimiento económico y progreso; pero, hoy en día se presenta como un proceso con una pluralidad de metas que van más allá de la rentabilidad (Faure et al. 2018, p.9).

En el caso de las innovaciones agrícolas, la rentabilidad de las inversiones está usualmente ligada a la corta duración de una etapa del ciclo biológico (como el sembrío de pastos mejorados o la inseminación de las vacas) (Ghadim et al., 2005, citado en Touzard, 2018, p. 53). Así, a diferencia de otros sectores económicos, las innovaciones agrícolas dependen fuertemente de las condiciones naturales. Las innovaciones agrícolas mantienen características específicas en su relación con la naturaleza, el espacio (tierras) y la sociedad (Touzard, 2018, p. 52).

Faure et al. (2018) resaltan que la innovación es un proceso complejo e impredecible. Precisamente, en las comunidades de Ocongate, además de la introducción de productos (lácteos) o procesos (transformación de la leche), también se han producido cambios no necesariamente esperados en la relación de las personas con su medio ambiente, familia y comunidad. Los mismos autores indican que los procesos de innovación requieren la sinergia de 3 dimensiones: la técnica, la organizacional y la institucional (Faure et al. 2018, pp. 12-13).

En el aspecto técnico, la innovación ha implicado cambios en el uso de las tierras, individuales y comunales, de Andamayo y Ccoñamuro; lo que incluye la introducción y/o perfeccionamiento de pastos cultivados destinados al ganado vacuno. Asimismo, se han introducido prácticas de buen manejo de los pastizales y ganado (como uso de cobertizos, mejoramiento del ganado, etc.). Tal vez lo más novedoso sea lo relacionado a la obtención de la leche cualificada para la elaboración de quesos y otros productos.

En la dimensión organizacional, la familia es el núcleo de la unidad agropecuaria. Los productores reconocen que los lazos familiares se han fortalecido, ya los trabajos temporales de los padres fuera de la comunidad se han reducido, y ahora pasan más tiempo con sus hijos. El papel de la comunidad como espacio de organización es reducido. La primera planta lechera de administración comunal que se estableció en el distrito funciona desde hace más de diez años la comunidad de Colca. Sin embargo, el resto de plantas transformadoras han sido implementadas por familias, que en muchos casos han dejado de criar ganado vacuno para dedicarse por completo a la producción de quesos. Esto sugiere que la gestión comunal no ha sido percibida como eficaz por los productores del distrito.

El aspecto institucional ha sido destacado por varios enfoques teóricos. El enfoque del Desarrollo Económico Local enfatiza el rol de los gobiernos locales, los que se plantean objetivos que corresponden a las circunscripciones donde se ubican, a diferencia de las instituciones de nivel nacional (Tello, 2008, p. 125). Por su parte, el 
Desarrollo Rural Territorial reconoce a la pluralidad de actores y procesos sociales que se articulan en un territorio, entre los que el Estado es uno más (Rojas, 2008). Esta congregación de esfuerzos posibilita el desarrollo local.

Las instituciones públicas y privadas ejecutaron diversos proyectos en Ocongate relacionados con la promoción agropecuaria y el fortalecimiento de capacidades en la producción y transformación de la leche de vaca, y su comercialización, entre otras actividades como la crianza de cuyes y la cosecha de hongos comestibles. Entre los organismos no gubernamentales destaca la prolongada presencia de la Asociación Jesús Obrero - CCAIJO, que forma especialistas en crianza de animales mayores y menores, forestación, explotación y transformación de hongos comestibles, panificación; hasta la formación de líderes. Asimismo, las obras de infraestructura de caminos, irrigación y materiales para la inseminación artificial del ganado fueron producto de la inversión de los gobiernos municipal y regional, los que cuentan con considerables presupuestos para la inversión en el desarrollo local.

Entre los productores que innovaron se ha hecho frecuente el término emprendedor para definirse. El término emprendedor se asocia a la independencia económica (ser su propio jefe), y a la superación de adversidades. Estos atributos modelan un estatus sobresaliente al que muchos quieren acceder. El anhelo se ve alimentado por el discurso sobre el emprendedor en el país, que lo reconoce como "[...] la base y el motor del crecimiento de un país" (Ministerio de la Producción, s.f., p. 8).

El emprendimiento no es una invención categórica nueva; aunque en la actualidad, tiene una connotación mercantil (Solís, 2017, p. 26). Una de las características comunes de las culturas humanas es que se adaptan constantemente a las necesidades y circunstancias de los seres humanos. Lo que caracteriza a los emprendedores modernos es que toman riesgos (Schumpeter, 1934). En este sentido, la innovación en agricultura involucra más riesgos que en otros rubros, debido a la imprevisibilidad del clima y la salud de los animales, por lo que muchas veces los agricultores pueden resistirse a adoptar cambios (Ghadim et al., 2005, citado en Touzard, 2018, p. 53).

Por último, debemos mencionar los posibles riesgos de las innovaciones llevadas a cabo en estas comunidades. En otros espacios altoandinos donde se ha introducido la ganadería de vacunos, Tapia (2008) resalta los impactos ambientales por el sobrepastoreo y el incremento de los hatos, entre otros. A nivel de la organización comunal para la tenencia de la tierra, en estas comunidades se viene dando la división y parcelación individual de los terrenos $(2008$, p. 475). Una situación que ya se presenta en las comunidades de Ocongate, pero que podría verse intensificada por la expansión de la ganadería familiar. Asimismo, podría verse intensificada la diferenciación social entre las familias de emprendedores y las que no han incursionado en la innovación.

\section{Conclusiones}

Las innovaciones en la cadena de producción de la leche de vaca han sido posibles por la sinergia en los ámbitos tecnológico, organizacional e institucional. 
Resaltan la toma de riesgos por parte de los productores, la provisión de las capacidades por organizaciones de cooperación internacional y la inversión de los gobiernos locales. El incremento en los ingresos familiares ha permitido cambios en las condiciones de vida de las familias y nuevos criterios de diferenciación social. Sin embargo, queda pendiente conocer cómo se están adaptando las familias de estas comunidades a los cambios económicos y sociales producidos por la pandemia de Covid-19.

\section{Agradecimientos}

El estudio se realizó gracias al apoyo del Convenio ARES-UNSAAC y del Fondo Especial de Desarrollo Universitario de dicha universidad. Agradecemos la asistencia de Celso Aimituma, José Pacco, Freinet Vera y Henry Cutiri en el recojo de datos, y los testimonios de la Asociación CCAIJO.

\section{Declaraciones del autor}

Los autores declaran que no hay conflictos de intereses en el presente artículo. 


\section{Referencias}

Aubron, C. (2014, julio). Ganadería Lechera: ¿una vía de desarrollo para los campesinos andinos? La Revista Agraria, 164. 10-11. http://www.larevistaagraria. org/content/ganader $\% \mathrm{C} 3 \% \mathrm{ADa}-1$ echera- $\% \mathrm{C} 2 \% \mathrm{BF}$ una-v $\% \mathrm{C} 3 \% \mathrm{ADa}-\mathrm{de}-$ desarrollo-para-los-campesinos-andinos.

Baregheh, A., Rowley, J., \& Sambrook, S. (2009). Towards a multidisciplinary definition of innovation. Management Decision, 47(8), 1323-1339. https://doi. org/10.1108/00251740910984578

Casallo, L.C. (2015). Surgimiento del emprendimiento campesino en el Distrito de Ocongate, Cusco. Aproximaciones desde la Experiencia [ponencia]. Seminario Internacional sobre desarrollo rural en la región andina amazónica. Arequipa. http://www.descosur.org.pe/wp-content/uploads/2016/06/Luis-Casallo.pdf

Faure, G., Chiffoleau, Y., Goulet, F., Temple, L. \& Touzard, J.M. (2018). Reviving perspectives on innovation in agriculture and food systems. En Innovation and development in agricultural and food systems (pp. 7-24). Editorial Quae.

Instituto Nacional de Estadística e Informática. (1993). Censos Nacionales: VIII de Población y V de Vivienda.

Instituto Nacional de Estadística e Informática. (1994). III Censo Nacional Agropecuario.

Instituto Nacional de Estadística e Informática. (2007). Censos Nacionales: XI de Población y VI de Vivienda.

Instituto Nacional de Estadística e Informática. (2012). IV Censo Nacional Agropecuario.

Instituto Nacional de Estadística e Informática. (2017). Censos Nacionales: XII de Población y VII de Vivienda.

Ministerio de la Producción. (s.f.). Emprendedor peruano. Oportunidades para el crecimiento empresarial. https://www.citepapa.pe/wp-content/uploads/2017/10/ EL-EMPRENDEDOR-PERUANO.pdf

Rojas López, J.J. (2008). La agenda territorial del desarrollo rural en América Latina. Observatorio de la Economía Latinoamericana, 96.

Rosas Morales, D. (2015). Productores Ganaderos, proyectos de desarrollo y poder: cambios en la orientación ganadera de Ocongate. En SEPIA (ed.) IV Censo Nacional Agropecuario. Resúmenes de investigaciones, experiencias y lecciones aprendidas. Programa de becas para jóvenes investigadores y tesis de maestría. Seminario Permanente de Investigación Agraria. (pp. 197-232). http://sepia.org. pe/facipub/upload/cont/1340/cont/files/CENAGRO\%20IV\%20SEPIA\%20 Mayo\%202015.pdf 
Schumpeter, J. (1934). The theory of economic development: an inquiry into profits, capital, credit, interest, and the business cycle. Transaction Books.

Solís, N. (2017). Los emprendimientos familiares: una aproximación desde la Antropología. Raíces. Revista Nicaragüense de Antropología 2(1). 26-32.

Tello, M. (2008). Desarrollo económico local, descentralización y clusters: teoría, evidencia y aplicaciones. CENTRUM-Católica.

Touzard. J.M. (2018). Agricultural and agrifood innovation in the 21st century: maintaining, erasing or reshaping its specificities? En Faure et al. (eds.) Innovation and development in agricultural and food systems (pp. 52-76). Editorial Quae.

Trivelli, C \& Hernández Asensio R. (2011). Crecimiento económico, cohesión social y trayectorias divergentes Valle Sur - Ocongate (Cuzco - Perú). Programa Dinámicas Territoriales Rurales RIMISP - Centro Latinoamericano para el Desarrollo Rural.

Tapia, M. (2008). La ganadería en el altiplano de Puno. Una visión técnica, económica, social y ambiental». En Damonte, G., Fulcrand, B. \& Gómez, R. (eds.) Perú: El problema agrario en debate - SEPIA XII (pp. 462-498). http://www.sepia.org.pe/ facipub/upload/cont/884/cont/files/02_sepia.pdf 